\title{
OPTIMAL BLOCK BASED DMT TRANSCEIVERS
}

\author{
See-May Phoong ${ }^{\dagger}$, and Yuan-Pei Lin ${ }^{\ddagger}$ \\ ${ }^{\dagger}$ Dept. of EE \& Grad. Inst. of Comm Engr.,National Taiwan Univ.,Taipei, Taiwan, R. O. C. \\ ${ }^{\ddagger}$ Dept. Elec. and Control Engr., National Chiao Tung Univ., Hsinchu, Taiwan, R. O. C.
}

\begin{abstract}
The design of optimal DMT transceivers for distorted channel with colored noise has been of great interest. Of particular interest is the class of block based DMT (BDMT), where the transmitter and the receiver consist of constant matrices. For a given channel and channel noise spectrum, the optimal DBMT transceiver that minimizes the transmission power for a fixed probability of error and transmission rate will be derived. The optimal biorthogonal transmitter is in fact orthogonal. That is, there is no loss of generality in using the orthogonal transmitter for the design optimal BDMT.
\end{abstract}

\section{INTRODUCTION}

There has been great interest in the design of DMT systems recently. Fig. 1 shows an example of an $M$-band DMT transceiver over channel $C(z)$ with additive noise $\nu(n)$. The example is the so-called block based DMT (BDMT), where the transmitter and the receiver consist of constant matrices. The encoding at the transmitter end and the decoding at the receiver end are done blockwise. The DMT is called orthogonal if the transmitter $\mathbf{G}_{0}$ in Fig. 1 is an orthogonal matrix, i.e., $\mathbf{G}_{0}^{T} \mathbf{G}_{0}$ is a diagonal matrix. We call it biorthogonal if $\mathbf{G}_{\mathbf{0}}$ is not orthogonal. When the receiver outputs are identical to the transmitter inputs in the absence of channel noise, the transceiver is said to be ISI free.

BDMT transceivers have been studied extensively. In the commonly used DFT based DMT, the transmitter and the receiver are DFT matrices [1]. In [2], more general orthogonal matrices are proposed. It is shown therein that, for AWGN (additive white Gaussian noise) frequency selective channels the optimal orthogonal transmitter consists of eigen vectors associated with the channel. In [3], optimal transceivers that minimize the total output noise power are developed. Information rate optimized DMT systems are considered in [4]. In [5], for a given distorted channel with colored noise the authors derive the optimal orthogonal transceiver that minimizes the transmission power for a fixed probability of error and transmission bit rate.

THIS WORK WAS SUPPORTED BY NSC 89-2213-E-002-122 AND 89-2213-E-009-118, TAIWAN, R.O.C.
Typical of DMT transmitters is the introduction of redundancy so that the receiver can cancel ISI due to the channel. Cyclic prefix, zero padding (or trailing zeros) and leading zeros are commonly used forms of redundancy. For example, cyclic prefix is used in DFT based DMT systems [1] and zero padding is considered in [2]-[5]. We will consider BDMT systems of trailing zeros only. In this paper, we will show that for any given transmission rate and probability of error, the optimal biorthogonal transceiver that minimizes the transmission power is orthogonal.

\section{ISI FREE BDMT WITH ZERO PADDING $[3,4]$}

Consider Fig. 1, where an $M$-band BDMT system is shown. Usually the channel is modeled as an LTI filter $C(z)$ with additive noise $\nu(n)$. Assume that $\nu(n)$ is a zero-mean WSS process and $C(z)$ is an FIR filter of order $L$ (a reasonable assumption after time domain equalization). So

$$
C(z)=c_{0}+c_{1} z^{-1}+\cdots+c_{L} z^{-L},
$$

with nonzero $c_{0}$ and $c_{L}$. The length of redundant samples is chosen to be $L$ so that the receiver can remove ISI due to $C(z)$ and decoding can be performed blockwise. Therefore the interpolation ratio $N$ is $N=M+L$. In the case of zero padding, the transmitter is of the form,

$$
\mathbf{G}_{0}=\left(\begin{array}{c}
\mathbf{G} \\
\mathbf{0}
\end{array}\right)
$$

where $\mathbf{G}$ is $M$ by $M$. Using this choice of $\mathbf{G}_{0}$, Fig. 1 can be redrawn as Fig. 2. The $N$ by $M$ matrix $\mathbf{C}$ is a lower triangular Toeplitz matrix whose first column is given by $\left[\begin{array}{lllllll}c_{0} & c_{1} & \ldots & c_{L} & 0 & \ldots & 0\end{array}\right]^{T}$. The $N \times 1$ vector $\nu$ shown in Fig. 2 is the blocked version of the scalar noise process $\nu(n)$. Using SVD, we decompose the channel matrix $\mathrm{C}$ as

$$
\mathbf{C}=\underbrace{\left(\begin{array}{ll}
\mathbf{U}_{0} & \mathbf{U}_{1}
\end{array}\right)}_{\mathbf{U}}\left(\begin{array}{c}
\boldsymbol{\Lambda} \\
\mathbf{0}
\end{array}\right) \mathbf{V}=\mathbf{U}\left(\begin{array}{c}
\boldsymbol{\Lambda} \\
\mathbf{0}
\end{array}\right) \mathbf{V}
$$

where $\mathbf{U}$ and $\mathbf{V}$ are orthonormal matrices of dimensions respectively $N \times N$ and $M \times M$, and $\Lambda$ is an $M \times M$ diagonal matrix. The biorthogonal DMT transceivers with ISI 


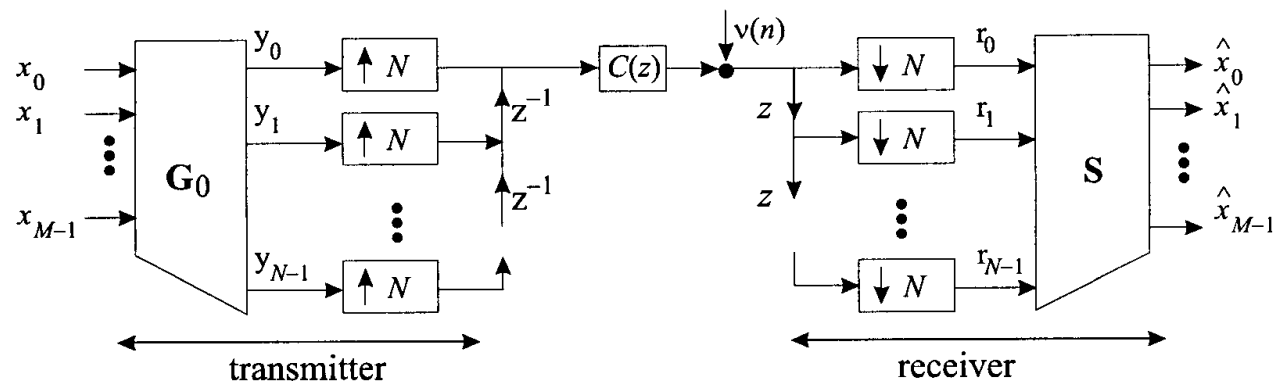

Figure 1: An $M$-band BDMT transceiver over channel $C(z)$ with noise $\nu(n)$.

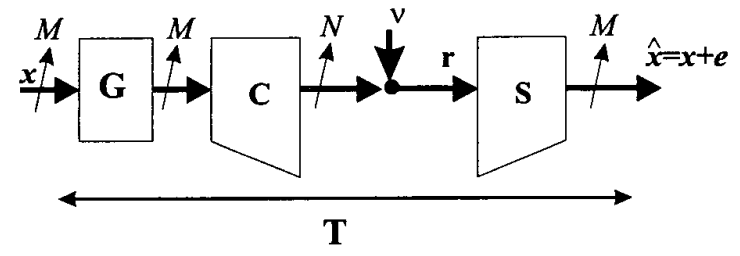

Figure 2: Matrix representation of the zero padded BDMT transceiver.

free property can be completely parameterized, as described in the following theorem.

Theorem 1 [3,4] The BDMT system with trailing zeros in Fig. I is ISI free if and only if the zero-padded transceiver satisfies the followings:

(i) $\mathbf{G}$ is an $M \times M$ nonsingular matrix;

(ii) $\mathbf{S}=\mathbf{G}^{-1} \mathbf{B}$, where $\mathbf{B}=\mathbf{V}^{T} \mathbf{\Lambda}^{-1}[\mathbf{I} \quad \mathbf{A}] \mathbf{U}^{T}$, for arbitrary $M \times L$ matrix $\mathbf{A}$.

\section{PROBLEM FORMULATION}

In this paper, we assume that the inputs $x_{k}$ are PAM symbols of $b_{k}$ bits. Without much loss of generality, we further assume that $x_{k}$ have zero mean and they are uncorrelated with each other. That is, $\mathcal{E}\left[x_{k} x_{m}\right]=\sigma_{x_{k}}^{2} \delta(k-m)$. This can always be obtained with proper interleaving. The average bit rate per symbol in this case becomes $b=\frac{1}{M} \sum_{k=0}^{M-1} b_{k}$.

The transmission power $P$ is the average energy of the vector $\mathbf{y}=\left(\begin{array}{llll}y_{0} & y_{1} & \cdots & y_{N-1}\end{array}\right)^{T}$ as shown in Fig. 1, $P=\frac{1}{N} \sum_{k=0}^{M-1} \sigma_{y_{k}}^{2}$. As the inputs $x_{k}$ are uncorrelated and have zero mean, $\sigma_{y_{k}}^{2}$ is given by $\sigma_{y_{k}}^{2}=\sum_{n=0}^{M-1}[\mathbf{G}]_{k n}^{2} \sigma_{x_{n}}^{2}$. Using this expression, we can write the transmission power as

$$
P=\frac{1}{N} \sum_{k=0}^{M-1} \sigma_{x_{k}}^{2}\left\|\mathbf{g}_{k}\right\|_{2}^{2}
$$

where $\left\|\mathbf{g}_{k}\right\|_{2}^{2}=\sum_{\ell=0}^{M-1}[\mathbf{G}]_{\ell k}^{2}$ is the energy of the $k$-th column of $\mathbf{G}$.

Under the ISI free condition, for a fixed bit rate and a fixed probability of error $P_{e}$, we will find the transceiver that minimizes the transmission power. The optimization process involves 2 steps. We will show in section 4 that the bits $b_{k}$ can be optimally allocated to minimize the transmission power for any given transceiver. Under the optimal bit allocation, the optimal transceiver will then be derived.

\section{OPTIMAL BIT ALLOCATION}

For a given transceiver, a fixed probability of error $P_{e}$, and average bit rate per input symbol $b$, we present the optimal bit allocation $\left\{b_{k}\right\}_{k=0}^{M-1}$ with $b=\frac{1}{M} \sum_{k=0}^{M-1} b_{k}$ such that the transmission power in (2) is minimized.

At the receiver end, the output of the $k$-th band is $\widehat{x}_{k}=$ $x_{k}+e_{k}$, where $e_{k}$ comes entirely from channel noise as the transceiver achieves zero ISI. Define the $M \times 1$ output noise vector as $\mathbf{e}=\left(\begin{array}{llll}e_{0} & e_{1} & \cdots & e_{M-1}\end{array}\right)^{T}$, then $\mathbf{e}=\mathbf{S} \boldsymbol{\nu}=$ $\mathbf{G}^{-1} \mathbf{B} \boldsymbol{\nu}$.

Assuming the PAM symbols of the $k$-th band carry $b_{k}$ bits, the probability of error for the $k$-th band is given by $P_{e}(k)=2\left(1-2^{-b_{k}}\right) Q\left(\sqrt{\frac{3 \sigma_{x_{k}}^{2}}{\left(2^{2 b_{k}}-1\right) \sigma_{e_{k}}^{2}}}\right)$. For a fixed probability of error $P_{e}$ across all bands, we need to have $P_{e}(0)=P_{e}(1)=\cdots=P_{e}(M-1)=P_{e}$. Under the high bit rate assumption $2^{b_{k}}-1 \approx 2^{b_{k}}$, we can see that $\sigma_{x_{k}}^{2}$ and $\sigma_{e_{k}}^{2}$ satisfy

$$
\sigma_{x_{k}}^{2}=c 2^{2 b_{k}} \sigma_{e_{k}}^{2}, \quad \text { where } \quad c=\frac{1}{3}\left(Q^{-1}\left\{P_{e} / 2\right\}\right)^{2} .
$$

Using this relation and applying the AM-GM inequality, the 
transmission power in (2) satisfies

$$
P \geq \frac{c M}{N} 2^{2 b} \prod_{k=0}^{M-1}\left(\sigma_{e_{k}}^{2}\left\|\mathbf{g}_{k}\right\|_{2}^{2}\right)^{1 / M} \triangleq P_{o p t, b i t} .
$$

The equality holds if and only if $2^{2 b_{k}} \sigma_{e_{k}}^{2}\left\|g_{k}\right\|_{2}^{2}$ are the same for all $k$. Notice that $P_{\text {opt,bit }}$ depends only on $b$ and $\sigma_{e_{k}}^{2}\left\|\mathbf{g}_{k}\right\|_{2}^{2}$, where $\sigma_{e_{k}}^{2}$ is determined once the receiver is known and $\left\|\mathbf{g}_{k}\right\|_{2}^{2}$ is determined once the transmitter is given. Therefore when the transceiver is given and average bit rate per symbol $b$ is fixed, $P_{\text {opt,bit }}$ is the lower bound of the transmission power independent of the bit allocation $\left\{b_{k}\right\}_{k=0}^{M-1}$. Solving for the optimal $b_{k}$, we have

$$
b_{k}=b-\log _{2}\left(\sigma_{e_{k}}\left\|\mathbf{g}_{k}\right\|_{2}\right)+\frac{1}{M} \log _{2}\left(\Pi_{\ell=0}^{M-1} \sigma_{e_{\ell}}\left\|\mathbf{g}_{\ell}\right\|_{2}\right) \text {. }
$$

The optimal bit allocation equalizes the terms $2^{2 b_{k}} \sigma_{e_{k}}^{2}\left\|\mathbf{g}_{k}\right\|_{2}^{2}$. When the transmitting vectors $\mathbf{g}_{k}$ have equal energy, we can see that more bits are assigned to bands where $\sigma_{e_{k}}^{2}$ is small. This is similar to the bit allocation in water filling scheme. More bits are transmitted in less noisy bands.

\section{OPTIMAL TRANSCEIVERS}

Optimal G: We first express the quantity $P_{o p t, b i t}$ in (4) in terms of $\mathbf{G}$. The energy of the $k$-th column of $\mathbf{G}$ is $\left\|\mathbf{g}_{k}\right\|_{2}^{2}=\left[\mathbf{G}^{T} \mathbf{G}\right]_{k k}$. Let $\mathbf{q}=\mathbf{B} \boldsymbol{\nu}$, then $\mathbf{e}=\mathbf{G}^{-1} \mathbf{q}$. The $M \times M$ autocorrelation matrix $\mathbf{R}_{e}$ of the noise vector $\mathbf{e}$ is given by $\mathbf{R}_{e}=\mathbf{S} \mathbf{R}_{\nu} \mathbf{S}^{T}=\mathbf{G}^{-1} \mathbf{R}_{q} \mathbf{G}^{-T}$, where $\mathbf{R}_{q}$ is the autocorrelation matrix of the vector $\mathbf{q}$. The output noise $\sigma_{e_{k}}^{2}$ of the $k$-th band is equal to $\left[\mathbf{R}_{e}\right]_{k k:}$ or $\left[\mathbf{G}^{-1} \mathbf{R}_{q} \mathbf{G}^{-T}\right]_{k k}$. So (4) can be rewritten as

$P_{o p t, b i t}=c \frac{M}{N} 2^{2 b}\left(\prod_{k=0}^{M-1}\left[\mathbf{G}^{T} \mathbf{G}\right]_{k k}\left[\mathbf{G}^{-1} \mathbf{R}_{q} \mathbf{G}^{-T}\right]_{k k}\right)^{1 / M}$

Apply the Hadamard inequality, we have

$$
\begin{aligned}
P_{o p t, b i t} & \geq c \frac{M}{N} 2^{2 b}\left(\operatorname{det}\left(\mathbf{G}^{T} \mathbf{G}\right) \operatorname{det}\left(\mathbf{G}^{-1} \mathbf{R}_{q} \mathbf{G}^{-T}\right)\right)^{1 / M} \\
& =c \frac{M}{N} 2^{2 b}\left(\operatorname{det} \mathbf{R}_{q}\right)^{1 / M} \triangleq P_{o p t, b i t, G}
\end{aligned}
$$

The equality holds if and only if (i) $\mathbf{G}^{\mathcal{T}} \mathbf{G}$ is diagonal and (ii) $\mathbf{G}^{-1} \mathbf{R}_{q} \mathbf{G}^{-T}$ is diagonal. The lower bound $P_{o p t, b i t, G}$ does not depend on the transmitter $\mathbf{G}$ and it is achieved if and only if $\mathbf{G}$ satisfies both conditions (i) and (ii). Let the Schur decomposition of $\mathbf{R}_{q}$ be

$$
\mathbf{R}_{q}=\mathbf{Q} \mathbf{\Sigma} \mathbf{Q}^{T}
$$

Then these 2 conditions can be satisfied by choosing $\mathbf{G}=$ $\mathbf{Q}$. The optimal transmitter $\mathbf{G}$ is orthogonal. In this case the receiver that achieves ISI is given by $\mathbf{S}=\mathbf{Q}^{T} \mathbf{B}$, where $\mathbf{B}$ is given in Theorem 1 .

Optimal A: From (6), we see that given any A, the achievable lower bound $P_{\text {opt }, \text { bit }, G}=c \frac{M}{N} 2^{2 b}\left(\operatorname{det} \mathbf{R}_{q}\right)^{1 / M}$. The matrix $\mathbf{A}$ should be chosen such that $\operatorname{det}\left(\mathbf{R}_{q}\right)$ is minimized. Using the facts that $\mathbf{R}_{q}=\mathbf{B} \mathbf{R}_{\nu} \mathbf{B}^{T}$ and $\mathbf{B}=$ $\mathbf{V}^{T} \Lambda^{-1}[\mathbf{I} \mathbf{A}] \mathbf{U}^{T}$, we get

$$
\operatorname{det}\left(\mathbf{R}_{q}\right)=\operatorname{det}\left(\boldsymbol{\Lambda}^{-2}\right) \operatorname{det}\left([\mathbf{I} \mathbf{A}] \mathbf{U}^{T} \mathbf{R}_{\nu} \mathbf{U}\left(\begin{array}{c}
\mathbf{I} \\
\mathbf{A}^{T}
\end{array}\right)\right) .
$$

Note that $\boldsymbol{\Lambda}, \mathbf{U}$ and $\mathbf{R}_{\nu}$ are fixed once the channel and input noise are given. Thus the optimal $\mathbf{A}$ is such that $\operatorname{det}\left(\left[\begin{array}{ll}\mathbf{I} & \mathbf{A}\end{array} \mathbf{U}^{T} \mathbf{R}_{\nu} \mathbf{U}[\mathbf{I} \mathbf{A}]^{T}\right)\right.$ is minimized. The optimal $\mathbf{A}$ has the following closed form expression (Readers are referred to [6] for detailed derivation).

$$
\mathbf{A}=-\mathbf{U}_{0}^{T} \mathbf{R}_{\nu} \mathbf{U}_{1}\left(\mathbf{U}_{1}^{T} \mathbf{R}_{\nu} \mathbf{U}_{1}\right)^{-1},
$$

where the matrices $\mathbf{U}_{0}$ and $\mathbf{U}_{1}$ are defined in (1). The minimum achievable $\operatorname{det}\left(\mathbf{R}_{q}\right)$ is

$$
\operatorname{det}\left(\boldsymbol{\Lambda}^{-2}\right) \operatorname{det}\left(\mathbf{R}_{\nu}\right) / \operatorname{det}\left(\mathbf{U}_{1}^{T} \mathbf{R}_{\nu} \mathbf{U}_{1}\right)
$$

Using this expression and (6), the minimum transmission power for the optimal transceiver is

$$
P_{\text {biortho }}=c 2^{2 b} \frac{M}{N}\left[\frac{\operatorname{det}\left(\mathbf{\Lambda}^{-2}\right) \operatorname{det}\left(\mathbf{R}_{\nu}\right)}{\operatorname{det}\left(\mathbf{U}_{1}^{T} \mathbf{R}_{\nu} \mathbf{U}_{1}\right)}\right]^{1 / M} .
$$

Summarizing the results, we have

Theorem 2 Consider the zero padded $M$-band DMT system in Fig. 2. Assume that the inputs are PAM symbols of $b_{k}$ bits. For any fixed probability of error $P_{e}$ and any fixed transmission bit rate per symbol b, the biorthogonal transceiver is ISI free and minimizes the transmission power $P$ in (2) if and only if the following are true:

(i) The matrix $\mathbf{A}$ is given by $\mathbf{A}=$ $-\mathbf{U}_{0}^{T} \mathbf{R}_{\nu} \mathbf{U}_{1}\left(\mathbf{U}_{1}^{T} \mathbf{R}_{\nu} \mathbf{U}_{1}\right)^{-1}$, where $\mathbf{R}_{\nu}$ is the autocorrelation matrix of the noise vector $\boldsymbol{\nu}$ and, $\mathrm{U}_{0}$ and $\mathrm{U}_{1}$ are as defined in (1).

(ii) The transmitter $\mathbf{G}=\mathbf{Q}$, where $\mathbf{Q}$ is the orthonormal matrix such that $\mathbf{Q}^{T} \mathbf{R}_{q} \mathbf{Q}$ is diagonal. The matrix $\mathbf{R}_{q}$ is given by $\mathbf{R}_{q}=\mathbf{B} \mathbf{R}_{\nu} \mathbf{B}^{T}$, where $\mathbf{B}=$ $\mathbf{V}^{T} \mathbf{\Lambda}^{-1}[\mathbf{I} \mathbf{A}] \mathbf{U}^{T}$. The receiver is given by $\mathbf{S}=\mathbf{Q}^{T} \mathbf{B}$.

(iii) The bits $b_{k}$ are allocated as $b_{k}=b-\log _{2}\left(\sigma_{e_{k}}\left\|\mathbf{g}_{k}\right\|_{2}\right)+$ $\frac{1}{M} \log _{2}\left(\prod_{\ell=0}^{M-1} \sigma_{e_{\ell}}\left\|\mathbf{g}_{\ell}\right\|_{2}\right)$.

The minimum transmission power is $P_{\text {biortho }}$ given in (9) 
Example. In this example we compare the transmission power of the derived optimal transceiver $P_{\text {biortho }}$, that of the orthogonal transceiver derived in [5] $P_{\text {ortho, }[5]}$, and that of the vector coding transceiver derived in [2] $P_{v c,[2]}$. The closed form expressions of $P_{\text {ortho, [5] }}$ and $P_{v c,[2]}$ are derived in [6]. The channel $C(z)$ and power spectrum for the colored noise $\nu(n)$ used in this example are showed in Fig. 3(a) and (b). These parameters are obtained from a typical ADSL environment. The channel $C(z)$ in this case has order $L=4$. The bit error rate $P_{e}=10^{-6}$ and average bit rate per sample is $R_{b}=\frac{M}{N} b=2$. The results are plotted for $M=10$ to 50 and they are showed in Fig. 4. One can see that the improvement of $P_{\text {biortho }}$ over $P_{\text {ortho, }[5]}$ is more significant when $M$ is small. When $M$ is large, the two curves converge. Also $P_{\text {biortho }}$ is approximately $5 \mathrm{~dB}$ smaller than $P_{v c,\{2\}}$. In the plot we see that $P_{\text {biortho }}$ and $P_{\text {ortho, } 5]}$ appear to be monotone decreasing. It is the authors' conjecture that $P_{\text {biortho }}$ and $P_{\text {ortho, [5] }}$ are monotone decreasing functions of $M$.

(a)
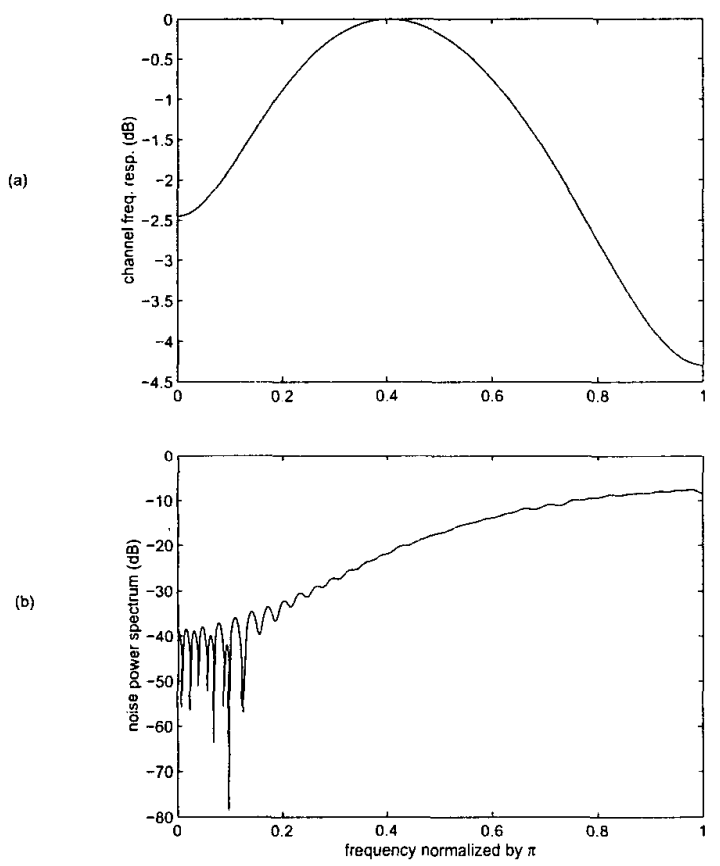

Figure 3: (a) The frequency response of the channel $\left|C\left(e^{j \omega}\right)\right|$. (b) The power spectrum of the channel noise $\nu(n)$.

\section{CONCLUDING REMARKS}

In this paper we show that, for the design of BDMT systems, there is no loss of generality in using orthogonal

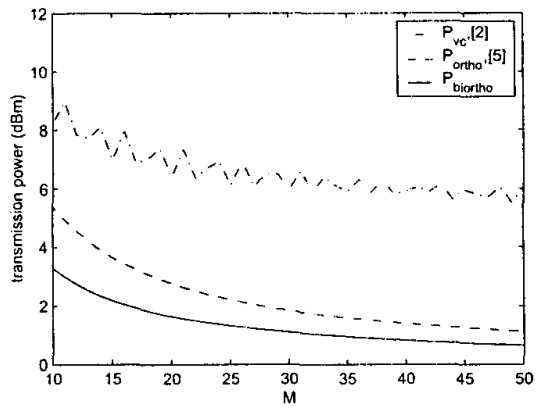

Figure 4: Performance comparison of $P_{\text {biortho, }} P_{\text {ortho, }[5]}$ and $P_{v c,[2]}$.

transceivers. The $M$-band DMT system is often considered as the dual of an $M$-band subband coder. The BDMT system can be considered as the dual of the transform coder. The result shown in this paper is similar to the result in subband coding theory that optimal orthogonal transform coder is as good as any biorthogonal transform coder [7].

\section{REFERENCES}

[1] P. S. Chow, J. C. Tu, and J. M. Cioffi, "Performance Evaluation of a Multichannel Transceiver System for ADSL and VHDSL Services," IEEE J. Sel. Areas Commun., pp. 909-919, Aug. 1991.

[2] S. Kasturia, J. T. Aslanis, and J. M. Cioffi, "Vector Coding for Partial Response Channels," IEEE Trans. $I T$, July 1990.

[3] A. Scaglione, G. B. Giannakis, and S. Barbarossa, "Redundant Filterbank Precoders and Equalizers Part I: Unification and Optimal Designs," IEEE Trans. SP, pp. 1988-2006, July 1999.

[4] A. Scaglione, S. Barbarossa, and G. B. Giannakis, "Filterbank Transceivers Optimizing Information Rate in Block Transmissions over Dispersive Channels," IEEE Trans. IT, pp. 1019-32, April 1999.

[5] Yuan-Pei Lin and See-May Phoong, "Perfect Discrete Multitone Modulation with Optimal Transceivers," IEEE Trans. SP, pp. 1702-11, June 2000.

[6] Yuan-Pei Lin and See-May Phoong, "Optimality of Orthogonal DMT Transceivers for Distorted Channels with Colored Noise," submitted to the IEEE Trans. SP.

[7] P. P. Vaidyanathan, "Theory of optimal orthonormal coder," IEEE Trans. Signal Processing, June 1998. 